\title{
Les courtiers locaux du développement
}

Jean-Pierre Olivier de Sardan et Thomas Bierschenk

\section{(2) OpenEdition}

Journals

Édition électronique

URL : http://journals.openedition.org/apad/3233

DOI : 10.4000/apad.3233

ISSN : 1950-6929

Éditeur

LIT Verlag

Édition imprimée

Date de publication : 1 juin 1993

\section{Référence électronique}

Jean-Pierre Olivier de Sardan et Thomas Bierschenk, « Les courtiers locaux du développement »,

Bulletin de l'APAD [En ligne], 5 | 1993, mis en ligne le 04 juin 2008, consulté le 08 septembre 2020. URL : http://journals.openedition.org/apad/3233 ; DOI : https://doi.org/10.4000/apad.3233

Ce document a été généré automatiquement le 8 septembre 2020

Bulletin de l'APAD 


\title{
Les courtiers locaux du développement
}

\author{
Jean-Pierre Olivier de Sardan et Thomas Bierschenk
}

1 Une proposition de recherche du SHADYC (Sociologie, Histoire, Anthropologie des Dynamiques Culturelles) dans le cadre d'une action concertée MRE/CNRS/ORSTOM "Problématiques de développement".

Contexte de la recherche

2 Parmi les pays du Sud, les pays africains sont, dans la conjoncture actuelle, ceux qui dépendent le plus de l'aide extérieure, proportionnellement à leurs ressources propres. Cette donnée économique est bien connue, mais elle a des implications proprement sociologiques moins visibles, liées aux modes de circulation et de redistribution de cette "rente du développement". En effet, toute perspective normative ou morale mise à part, il s'agit bien là d'une "rente", même si cette rente opère dans un contexte de misère et se fonde, à la différence des rentes minières, sur la mobilisation de ressources externes. Dire, par exemple que l'État africain est un État assisté, est une façon de dire que C'est un Etat rentier. Mais, et c'est une des facettes de la "crise de l'Etat en Afrique", les Etats africains contemporains ne sont pas en mesure de drainer ou de contrôler une grande partie des flux Nord-Sud. La "rente du développement" transite donc pour beaucoup par des réseaux d'intermédiaires nationaux, qui ne se confondent pas avec les appareils administratifs et politiques classiques. L'importance croissante des ONG comme opérateurs de développement en témoigne, de même que la place prise par le système des "projets", y compris dans les coopérations bilatérales et multilatérales. De ce fait les interlocuteurs locaux prennent de plus en plus d'importance.

Définition

3 Par "courtiers locaux du développement", nous entendons les acteurs sociaux implantés dans une arène locale qui servent d'intermédiaires pour drainer (vers l'espace social correspondant à cette arène) des ressources extérieures relevant de ce que l'on appelle communément "l'aide au développement". Si l'on prend le "projet de développement" comme la forme quasi idéal-typique de l'opération de développement 
aujourd'hui, quel qu'en soit l'opérateur, les courtiers représentent les porteurs sociaux locaux d'un projet, ceux qui constituent l'interface entre les destinataires du projet et les institutions de développement, ceux qui sont censés représenter la population locale (ou en exprimer les "besoins") vis à vis des structures d'appui et de financement extérieure.

Problématique de recherche

Les courtiers du développement ne tombent pas du ciel. Ils sont le produit d'histoires locales, et fonctionnent à l'intérieur de réseaux. C'est à connaître les processus sociaux qui constituent certains acteurs africains en courtiers du développement, que cette recherche est consacrée. La mobilisation ou la captation de ressources extérieures par ces courtiers, au profit de groupes ou collectivités au nom desquels ils entendent agir et pour lesquels ils se positionnent comme mandataires, ne peut évidemment se réduire aux motivations affichées, qui relèvent de diverses rhétoriques du bien public, de l'intérêt collectif, du dévouement aux autres, du militantisme développementiste... Il s'agit aussi de pouvoir ou d'influence, si ce n'est d'intérêts plus matériels. Les courtiers tentent donc, au-delà des idéologies qu'ils revendiquent, de renforcer leur position dans l'arène locale (et parfois dans l'arène nationale). Un lien s'établit alors entre le courtage en développement et les systèmes clientélistes souvent signalés (bien que peu étudiés empiriquement). Mais l'influence des courtiers n'est pas seulement due à leur contrôle plus ou moins étendu et plus ou moins direct des modes d'affectation ou de redistribution des ressources que l'aide au développement permet de drainer vers le village, le quartier, la région, il dépend aussi de leur capacité de négociation et de partenariat avec les opérateurs du Nord, et donc de leur insertion personnelle dans des réseaux Nord-Sud plus ou moins institutionnalisés. Une condition de cette insertion semble être leur compétence rhétorique, c'est-à-dire leur capacité à s'exprimer dans un langage approprié aux attentes des institutions de développement et des bailleurs de fond. Il s'agit d'un savoir-faire évolutif, qui doit leur permettre de s'adapter aux nouvelles "modes" du développement, qui surgissent régulièrement ("besoins fondamentaux", "sustainable development", "auto-promotion", etc...). La "localisation" croissante des projets (le fait que de plus en plus les circuits étatiques verticaux classiques soient court-circuités au profit d'une aide arrivant "directement" aux destinataires) aboutit paradoxalement à multiplier le nombre des courtiers locaux ("à la base", à l'échelle $d u$ village ou $d u$ quartier), tout en impulsant l'"internationalisation" de ces courtiers (reliés désormais directement et régulièrement à des partenaires européens ou nord-américains).On voit ainsi se dessiner les trois grands axes de notre travail :

La typologie des courtiers et de leurs réseaux d'appartenance

Un premier inventaire fera ainsi apparaître quatre grandes catégories :

6 - Les réseaux" confessionnels"

7 L'appartenance à une église, une secte, une confrérie, permet la mobilisation de contacts sociaux extérieurs à la communauté locale et aux appartenances lignagères et familiales. Il s'agit sans doute, avec les entreprises missionnaires d'antan et les organisations caritatives d'aujourd'hui, des premières formes de courtage décentralisé. Le thème particulièrement intéressant de "religion et développement" peut ainsi être abordé non pas par le biais classique des "idéologies religieuses" mais par celui des espaces sociaux que tissent les institutions religieuses, et la position d'intermédiaires que certains occupent en leur sein. Confréries islamiques, Eglise catholique, églises et 
sectes protestantes, mouvements syncrétiques, sont autant de réseaux par lesquels des courtiers inscrits dans ces institutions respectives mobilisent de l'aide au développement. universitaires, immigrés, commerçants) issus d'une même région, d'un même bourg, d'un même village, se sont multipliées en Afrique ces dernières années, et impulsent des projets de développement de plus en plus nombreux, en s'appuyant sur leurs compétences professionnelles, comme sur leurs relations sociales ou politiques acquises en ville ou à l'extérieur. Par là ils gardent ou retrouvent non seulement un lien avec leur origine sociale, mais aussi ils prennent pied dans l'arène politique locale.

10 - Les mouvements culturels/ethniques

11 Souvent animés par des fonctionnaires ou des intellectuels, ces mouvements tentent à la fois de faire bénéficier les populations dont ils se réclament d'une plus grande part de la rente de développement, au nom en général d'inégalités dont ils s'estiment victimes dans la répartition "ethnique" de cette rente, et d'obtenir pour leurs dirigeants un meilleur accès aux positions politiques. Si l'ethnicité à fait l'objet d'innombrables travaux, les mouvements ethniques, largement réactivés ou suscités par le contexte actuel de la démocratisation, ont peu fait l'objet d'études de terrain : les aborder sous l'angle des processus de médiation entre populations et Etat, de captation/redistribution des flux d'aide, de renforcement des positions politiques et des capacités clientélistes, en un mot, du point de vue du courtage en développement, semble une perspective particulièrement riche (que l'on pense au problème touareg vu sous cet angle...).

12 - Les "leaders paysans"

13 Cette expression, employée par de nombreuses ONG et agences de développement, entend désigner les partenaires issus du milieu agricole que l'on tente de former et avec lesquels on entend traiter, autrement dit ceux que les institutions du Nord tentent d'ériger en position de "courtiers aux pieds nus"... Certains ont ainsi acquis un savoir-faire important qui leur permet de traiter directement avec les bailleurs de fond. L'ajustement structurel, d'autre part, renvoie de plus en plus de scolarisés vers les campagnes, qui eux aussi, avec ce "retour à la terre", cherchent à occuper la position d'interlocuteurs paysans des institutions de développement. Les structures dites "collectives" (coopératives, groupements paysans, associations villageoises de développement) constituent un des lieux d'émergence de ces leaders paysans (et d'affrontements éventuels avec les notables locaux).

Ces catégories ne sont pas nécessairement exclusives entre elles, pas plus qu'elles n'excluent d'autres formes de courtage local. Mais elles semblent être les plus significatives, et les plus intéressantes en termes de recherche comparative. Elles posent en effet clairement les questions auxquelles nous tenterons de répondre dans les deux derniers axes de travail.

Les relations entre courtiers locaux en développement et médiateurs traditionnels

15 Nombre des pouvoirs locaux de type traditionnel, chefs de lignage, chefs de village, chefs de cantons, "notables", avaient et ont encore parfois une fonction de médiation. La colonisation, on le sait, a ainsi recouru, tant pour son administration que pour sa "mise en valeur" (ancêtre du développement) à ces intermédiaires indigènes qu'elle 
n'hésitait pas à introniser dans des fonctions de représentation politique traditionnelle ou pseudo-traditionnelle. Si ces médiateurs ont en leur temps bénéficié (et bénéficient parfois encore) de diverses rentes de position (redevances, prébendes ou détournements), ils sont souvent à l'écart de la rente de développement. En particulier, à la différence des courtiers en développement, ils ne maîtrisent pas les discours propres au monde du développement. Les rapports entre médiateurs politiques classiques et courtiers en développement ne sont cependant pas simples : ils traversent en effet des appartenances familiales, sociales ou ethniques, et se superposent avec de multiples conflits ou alliances locales. Ne peut-on pas cependant déterminer l'apparition d'une sorte de "compromis historique" entre les médiateurs traditionnels gérant les rapports entre Etat et acteurs locaux, et les courtiers en développement, gérant les rapports entre institutions de développement et acteurs locaux?

Les nouveaux clientélismes

On a depuis longtemps identifié l'existence de formes "néo-patrimoniales" en Afrique.

Mais l'émergence des courtiers en développement ne suscite-t-elle pas de nouveaux rapports de patronage, basés sur la capacité du courtier à orienter ou redistribuer les flux de l'aide vers tel ou tel, alimentant ainsi des relations d'allégeance? Doit-on y voir la résurgence de formes plus anciennes de clientélisme (par exemple réactivation de liens de dépendance personnels récurrents entre deux familles), ou bien les "règles du jeu" développementiste ne permettent-elles pas l'émergence de nouveaux modes d'affiliation (liés au caractère nouveau des ressources mobilisées par les courtiers: relations avec les bailleurs de fond, capital scolaire minimum, insertion dans des réseaux extérieurs)?

État des lieux

17 Si l'approche des phénomènes sociaux de développement en termes de "courtage" nous semble originale, elle ne tombe cependant pas du ciel, et nous devons reconnaittre notre dette auprès de quatre courants d'analyse :

18 1) L'anthropologie africaniste anglo-saxonne : autour de l'École de Manchester, le rôle politique moderne de la chefferie a été bien mis en valeur, en particulier sa fonction de médiation (analyse de Gluckman, reprises par Balandier et autres: Gluckman, 1949; Balandier, 1967).

19 2) La sociologie rurale européenne : elle a aussi insisté de son côté, avec Mendras (1976), sur la position de médiateur entre la société locale et la société englobante tenue par les notables ruraux, et sur l'importance de ces derniers quant à l'innovation.

3) Norman Long (1989) : lui aussi issu de l'École de Manchester, il a attiré l'attention sur la notion d'interface, au sens d'interface entre populations locales et institutions de développement, comme lieu stratégique de l'analyse des logiques sociales, et des rapports de pouvoir consubstantiels à la pratique du développement.

21 4) La science politique africaniste française : avec la revue Politique Africaine, elle a attiré l'attention sur les fonctions néo-patrimoniales de l'Etat (Médart, 1991), son rôle prédateur et rentier et son caractère extraverti (Bayart, 1989), et l'importance de phénomènes tels que le clientélisme, la corruption, l'économie informelle, etc...

22 D'autre part, nous avons développé autour du GRA, Groupe de Recherche Anthropologique à Marseille (aujourd'hui inclus dans le SHADYC, CNRS-EHESS) depuis plusieurs années, une réflexion autour des différents problèmes de l'anthropologie du développement, qui a débouché de plus en plus sur un point de vue relevant de ce que 
l'on pourrait appeler une micro-anthropologie politique du développement: les faits sociaux de développement doivent être saisis à l'échelle locale, parce que c'est là que se confrontent les multiples stratégies des acteurs, et les logiques sociales et culturelles qui les informent ; la confrontation qui s'opère est un phénomène d'ordre politique, car les ressources en pouvoir sont prépondérantes, comme enjeux autant que comme atouts (cf. Bierschenk, 1988; Bouju, 1991; Olivier de Sardan, 1984). Cette approche "micro" n'est en aucun cas une fuite dans l'accumulation obsessionnelle d'études de cas, ou l'oubli des contraintes de,type "macro": elle se réfère plutôt d'un coté à la "extended case study method" de l'Ecole de Manchester (dans laquelle nous serions tenté de voir l'ancêtre direct et méconnu de la micro-storia italienne, dont la démarche nous semble également pertinente quant à notre objet), et d'un autre coté à la conception de l'agencéité (agency) selon Giddens (cf. Giddens, 1979), autrement dit la recherche des marges de manœuvre de l'acteur en situation sociale semi-contrainte.

La double dimension empirique (analyses de terrain) et comparative (construction de typologies) nous semble, faut-il le préciser, indispensable: c'est d'ailleurs là une orientation fondamentale du laboratoire SHADYC.

Réseaux d'appui

Dans le cadre du DEA "Sciences sociales" de l'EHESS à Marseille, nous avons depuis plusieurs années mis en place une filière sur l'anthropologie du développement en Afrique, avec actuellement douze doctorats en cours relevant de cette filière (et un flux d'une dizaine de DEA par an, dont la moitié venant d'Afrique).

Le SHADYC entretient d'autre part des liaisons étroites avec le Laboratoire d'Etudes Agraires de l'ORSTOM à Montpellier, et avec le GDR 946 (Anthropologie comparée des sociétés de la Boucle du Niger), dans le cadre duquel a été menée une recherche ASP-CNRS-ORSTOM.

Toujours autour du SHADYC à Marseille s'est développée depuis deux ans l'APAD. L'association nous permet, d'un côté, de repérer les chercheurs africains intéressés par cette problématique, soit qu'ils aient déjà des matériaux pour alimenter de façon empirique le débat, soit qu'ils entendent mener des recherches de terrain sur ce thème. D'un autre coté, le Bulletin pourra servir à faire circuler les contributions à la recherche sur "les courtiers locaux du développement" dans l'ensemble du réseau, et ainsi alimenter un véritable débat scientifique sur cette question, à une échelle non négligeable.

Dispositif de recherche

Étant donné que tous les chercheurs en anthropologie du développement ont déjà des données sur le thème des "courtiers locaux en développement", même s'il n'a jamais fait l'objet de recherches systématiques (il intervient à la marge de toute étude, et tout chercheur est lui-même sollicité...) il s'agit surtout d'organiser, de systématiser et de compléter ces données.

Si les recherches de complément peuvent se faire à l'échelle individuelle, les aspects d'élaboration de la problématique et de la méthodologie sont centraux, et le programme comporte un aspect important de recyclage, d'élaboration théorique et de formation : la venue en France de nos collègues africains pour des séjours de 3 mois en prend d'autant plus d'importance. Quant aux missions en Afrique des chercheurs français, elles devront comporter une part importante d'encadrement des recherches de terrain pour les doctorants et de travail de séminaire sur place. Les deux ateliers 
prévus seront coordonnés avec ces déplacements. Les coordinateurs du projet seront J.-P. Olivier de Sardan et T. Bierschenk, avec la participation des chercheurs du SHADYC.

Objectif

L'objectif du présent programme est la publication d'un ouvrage collectif sur "les courtiers locaux du développement" (fin 1995). Pour la sélection des contributions à cet ouvrage, un comité de lecture international sera constitué. Les membres de ce comité participeront également au séminaire international final.

Calendrier

Le programme se déroulera en plusieurs étapes en 1994 et 1995.

1er trimestre 1994

Organisation d'un atelier de recherche regroupant tous les participants de l'équipe (chercheurs français, étrangers, doctorants) pendant une semaine à Marseille, afin de développer et affiner la problématique.

2ème et 3ème trimestres 1994

Recherches individuelles sur le terrain (2 à 3 mois).

4ème trimestre 1994

Dépouillement et analyse des données.

Organisation d'un atelier de recherche regroupant tous les participants de l'équipe (chercheurs français, étrangers, doctorants) à Marseille, afin de comparer les résultats des recherches individuelles.

Rédaction des rapports de recherche

Lancement d'un appel à contributions pour un séminaire international.

1er et 2ème trimestres 1995

Organisation d'un séminaire international. Il pourrait être "logé" dans le cadre du congrès biennal d'une société savante ou d'une association professionnelle (par exemple APAD, ou Association Européenne des Anthropologues Sociaux, ou Association Européenne de Sociologie Rurale...).

3ème et 4ème trimestre 1995

Rédaction des contributions à un ouvrage collectif basé sur les études entreprises dans le cadre du présent programme et les contributions au séminaire international.

\section{AUTEURS}

JEAN-PIERRE OLIVIER DE SARDAN

Marseille 


\section{THOMAS BIERSCHENK}

Berlin 